\title{
State-of-the-Art of Monoclonal Antibodies for the Treatment of Gastric Cancer
}

\author{
Debora Basile' \\ Francesca Simionato' \\ Alessandro Cappetta (iD) \\ Silvio Ken Garattini ${ }^{2}$ \\ Giandomenico Roviello ${ }^{3}$ \\ Giuseppe Aprile' \\ 'Department of Oncology, San Bortolo \\ General Hospital, AULSS8 Berica, \\ Vicenza, Italy; ${ }^{2}$ Department of Oncology, \\ University Hospital, Udine, Italy; \\ ${ }^{3}$ Department of Health Sciences, Section \\ of Clinical Pharmacology and Oncology, \\ University of Firenze, Firenze, Italy
}

Correspondence: Giuseppe Aprile

Department of Oncology, AULSS8 Berica,

Vicenza, Italy

Email giuseppe.aprile@aulss8.veneto.it

\begin{abstract}
Gastric cancer (GC) is a complex and heterogeneous disease with poor prognosis and limited available treatment options. During recent years, several molecular stratifications have been proposed to optimize the overall treatment strategy for GC patients. Breakthroughs in cancer biology and in molecular profiling through DNA and RNA sequencing are now opening novel landscapes, leading to the personalization of molecular matched therapy. In particular, therapies against HER2, Claudine 18.2, Fibroblast Growth Factor Receptors (FGFR), and other molecular alterations could significantly improve survival outcomes in the advance phase of the disease. Furthermore, immunotherapy with checkpoint inhibitors also represents a promising option in a selected population. Hoping that precision oncology will enter soon in clinical practice, our review describes the state of the art of many novel pathways and the current evidence supporting the use of monoclonal antibodies implicated in GC treatment.
\end{abstract}

Keywords: gastric cancer, precision oncology, HER2-inhibitors, Claudine 18.2 inhibitors, FGFR inhibitors

\section{Introduction}

Gastric cancer (GC) represents the fifth common tumor and the third leading cause of cancer-related death globally since it frequently occurs at an advanced stage at diagnosis. ${ }^{1}$ For these patients, the mainstay of the treatment is still represented by systemic chemotherapy based on platinum- and fluoropyrimidine combination. Of note, GC is a heterogeneous disease that arises from multiple interplay of genetic, environmental, and host characteristics, in which initiation, cancer growth, and metastatic spreading result from several genomic alterations that induce the activation of different molecular pathways. Although the increasing number of target therapy options in several cancer types, the target treatments in GC are limited, and current options for selected patients include HER2 blockade, anti-VEGF and immunotherapy. Historically, the increased knowledge on potential antigen targets expressed on cancer cells has stimulated the use of monoclonal antibodies to tackle cancer progression. The killing capabilities of these molecules may result from direct action of the antibody, immune-mediated mechanisms including complement-dependent and antibody-dependent citotoxicities, payload delivery, specific inhibitory effect of the antibody on targets expressed on the neovascular tumor or the surrounding stroma, or a combination of these antineoplastic actions. With time, an increasing number of monoclonal antibodies have received approval from the US Food and Drug Administration for the treatment of various solid tumors and 
hematological malignancies. Nowadays, the use of monoclonal antibodies has become routinary and has achieved significant clinical success in many cancer types. Despite the proven efficacy of these treatments, the prognosis of advanced gastric cancer remains poor with a median survival of approximately 12 months. ${ }^{2-6}$ Despite considerable efforts to improve comprehensive molecular classifications for GC focused at offering a precision strategy to patients, only a few drugs have been currently approved. In the present review, we discuss the updates about molecular features and promising target agents that could facilitate precision medicine on GC in the near future.

\section{Novel Hint on the Molecular Profile in Gastric Cancer}

To date, molecular classification of GC is a complex topic of discussion that we have extensively discussed elsewhere. $^{7}$ In brief, Lauren and World Health Organization (WHO) classifications are the most common used during pathological assessment. Lauren classified GC into intestinal, diffuse, and mixed histology. The most frequent subtype is intestinal with liver organotropism. Conversely, diffuse-types are locally aggressive with high rates of peritoneal invasion. ${ }^{8,9}$ WHO stratification proposed a division into tubular, papillary, mucinous, poorly cohesive (including Lauren diffuse type), and mixed histology.

Despite their advantage in clarifying putative histological origin, neither classification is currently useful in guiding real world treatment strategies. In recent years, the molecular characterization of GC is rapidly evolving, with novel insights in molecular findings that allow the identification of targetable pathways. The Cancer Genome Atlas (TCGA) suggested a comprehensive molecular stratification of GC according to genomic, transcriptomic and proteomic data into four different subtypes: tumors with chromosomal instability (CIN), genomically stable (GS) cancers, Epstein-Barr Virus (EBV)-positive tumors and microsatellite instability (MSI) tumors. ${ }^{10}$

Tumor tissues of 295 early GC patients were analyzed. More than $50 \%$ of GC belong in CIN subgroup, which display receptor-tyrosine kinase (RTK/RAS) pathway activation, amplification of ERBB2, ERBB3, EGR, FGFR, MET, KRAS/NRAS and cyclins responsible of cell divisions. GS tumors are associated with mutations of CDH1 and RHOA or CLDN18-ARHGAP fusion. EBV-positive tumors (approximately 9\%) are enriched for mutation in PIK3CA and
ARIDIA, extreme DNA hypermethylation, and high amplification of PD-L1, PD-L2 and JAK2. MSI high tumors (22\%) are characterized by high mutation rates accumulating alterations in PIK3CA, ERBB3, HER2 and EGFR. ${ }^{10}$

In 2015, The Asian Cancer Research Group (ACRG) developed a classification with 4 molecular subgroups based on array-based gene-expression profiling: MSI-H, microsatellite stable with epithelial-to-mesenchymal transition features (MSS/ EMT), MSS/TP53 mutant (MSS/ TP53), and MSS without TP53 mutation (MSS/TP53-). ${ }^{11}$

Intriguingly, MSI-H and EBV-positive subtypes, display high inflammatory infiltrate of TCD $8+$ cells and that could impact on immune treatment. ${ }^{12-15}$

However, current molecular evaluation based on tissue analysis is unable to capture spatial and temporal tumor heterogeneity, clonal evolution, and secondary resistances due to therapeutic strategies. Noteworthy, discordance between primary tumor and metastatic lesions is observed in $32 \%$ of advanced GC; while $87.5 \%$ is the concordance for mutation between metastatic tumor and circulating tumor DNA (ctDNA). ${ }^{16,17}$ Therefore, in the near future, liquid biopsy assessing circulating cancer-derived molecules could optimize treatment selection. Of note, liquid biopsy could exert its role in GC in several manner according setting. Firstly, ctDNA plays a key role in the early detection of metastatic disease and estimation of tumor volume: a subset analysis of $18 \mathrm{GC}$ patients showed an association between high ctDNA level and recurrence. The role in detecting molecular residual disease has been prospectively evaluated in 46 resected GC patients with stage I-III. Presence of ctDNA was reported in $45 \%$ of samples and independently associated with tumor stage and recurrence; interestingly, ctDNA positivity has been detected approximately 6 months before recurrence. Moreover, in recent years ctDNA evaluation has been demonstrated as a promising analysis for response monitoring and to follow treatment resistance. Wang et al showed that ctDNA predicted anti-cancer response in $24 \mathrm{GC}$ patients treated with trastuzumab, observing a decreased HER2 copy number in progressing patients.

\section{Targeting HER2: From Trastuzumab to Novel Compounds}

Epidermal growth factor receptor (EGFR, also called ERBB or HER) is a membrane tyrosine kinase receptor composed by an intracellular, a transmembrane lipophilic and a cysteine-rich extracellular ligand-binding domain. Amplification or over-expression of HER2 plays a key 
role in cancer initiation and progression, through activation of several signaling pathways like PI3K/AKT/mTOR. HER2-EGFR dimers, HER2 homodimers and HER2HER3 dimers promote cancer growth by sustaining tumor cell metabolic functions, cell survival, proliferation and metastatic spreading. Interestingly, HER2 protein has become a therapeutic target in different cancer types. Several monoclonal antibody with anti-HER2 functions have been developed and evaluated in several tumors including GC, where it accounts for approximately 6 $30 \%$ of all cases. ${ }^{18,19}$ HER2-positive GC is mostly detected in intestinal subtype, located at proximal tumor site, as high tumor grade, and they are usually well- or moderately-differentiated. Nonetheless, HER2 inhibition in GC has not achieved the outstanding results reported in breast tumors. ${ }^{20}$ The anti-HER2 antibody trastuzumab has been the only well established cornerstone for many years in GC treatment. The Phase III TOGA trial assessed trastuzumab-containing regimen compared with standard first-line chemotherapy. Trastuzumab yielded a statistical improvement in terms of median overall survival (OS), (13.8 months for trastuzumab containing regimen vs 11.1 months for standard arm; HR 0.74, 95\% CI 0.60-0.9, $\mathrm{P}=0.0046)$, median progression-free survival (PFS) $(6.7$ months vs 5.5 months, respectively; $\mathrm{P}=0.0017$ ) and overall response rate (ORR) $(47 \%$ vs $35 \%$, respectively; $\mathrm{P}=0.0017) .{ }^{21}$ Henceforth, based on these results trastuzumab combination therapy became the gold standard for advanced HER2-positive GC. ${ }^{21}$ Since these data, many attempts have been made to improve survival outcomes in this cohort of GC patients by assessing many other HER2-targeting molecules. Lapatinib, a potent ATP-competitor of both EGFR and HER2 tyrosine kinase inhibitor, failed to demonstrate OS improvement in the first-line phase III TRIO-013/LOGIC trial comparing capecitabine and oxaliplatin with or without lapatinib (12.2 months versus 10.5 months, respectively; HR 0.91 ; 95\% CI $0.73-1.12){ }^{22}$ In second-line phase III TYTAN trial, the addition of lapatinib to paclitaxel showed no benefit on survival outcomes. Noteworthy, in the subgroup analysis, tumors with $\mathrm{IHC} 3+$ score was associated with better OS ( 14.0 vs 7.6 months; HR $0.59, \mathrm{P}=0.02$ ), conversely no difference was reported in IHC2+ tumors. ${ }^{23}$ Based on promising results in advanced HER2-positive breast cancer, another recombinant humanized monoclonal antibody that exerts its action through the dimerization of HER2 with other HER receptors has been examined. Namely, the phase III JACOB trial evaluated the combination of pertuzumab and trastuzumab with chemotherapy in 780 HER2-amplified GC patients candidate to receive firstline treatment. ${ }^{24}$ Despite the 3.3 month improvement in terms of median OS in the experimental arm, no statistical significant benefit has been reached (14.2 vs 17.5 months; HR 0.84, 95\%C.I. 0.71-1.00; $\mathrm{P}=0.057$ ). Notably, significant improvement on PFS has been observed (8.5 vs 7.0 months; HR 0.73, 95\%C.I. 0.86; $\mathrm{P}=0.0001){ }^{23}$

Trastuzumab emtansine (T-DM1), an antibody-drug conjugate consisting of trastuzumab with the tubulin inhibitor emtansine, was also tested as second-line versus physician's choice in patients progressing after first-line trastuzumab based therapy in the phase II/III GATSBY trial. $^{25}$ Patients were randomized to receive taxane or T-DM1. The experimental anti-HER2 agent did not prolong OS and PFS compared with standard arm (7.9 versus 8.6 months, HR 1.15, CI 0.87-1.51, P =0.86). ${ }^{25}$ Similar results have been obtained when trastuzumab was tested in combination with paclitaxel beyond progression in Phase II trials in patients resistant to first-line with trastuzumab and platinum-based treatment. ${ }^{26}$

These contradictory results suggest the intrinsic differences in the HER2 biology between GC and breast cancer cells, which reflects the heterogeneous pattern of expression in GC (that usually ranges from 26 to $79 \%$ in IHC) affecting response to anti-HER2 treatments. This becomes evident especially in the second-line setting due to loss of dependence on HER2 biology during first-line treatment because of pharmacological pressure ${ }^{27-29}$ Several potential mechanisms of resistance could explain the lack of benefit of anti-HER2 blockade. In particular, $\mathrm{GC}$ is a complex heterogeneous disease. In particular, the HER2 membranous expression in GC is mainly basolateral and incomplete linked to a high intratumoral heterogeneity. Moreover, HER2 distribution is different between primary tumor and metastatic lesions. As a result, current guidelines recommend to test a minimum of 5 tumor biopsies to confirm HER2 positivity. ${ }^{30}$ Notably, the VARIANZ study conducted on 548 GC patients enrolled investigated the concordance between central and local assessment of HER2 test. Final 77 HER2 positive GC were confirmed by central assessment with a deviation rate of approximately $22 \%$, especially in borderline HER2 positive GC. Patients treated with trastuzumab and with HER2 confirmed GC by central evaluation were associated with longer survival than patients receiving trastuzumab according local assessment (20.5 months vs 10.9 months; HR 0.42 ; 95\% CI, 8.2 to 14.4 ; $P<0.001$ ). 
HER2 positivity detected in $\geq 40$ cancer cells and a HER2 amplification ratio of $\geq 3.0$ have been proposed as the optimal thresholds to identify patients benefiting from trastuzumab-based therapy.

Moreover, the presence of emerging molecular alterations, such as PI3K, MAPK activation and MET or FGFR aberrations are involved in the resistance to HER2directed agents. ${ }^{16,31-34}$

Noteworthy, a pan-HER inhibitor afatinib, that irreversibly blocks EGFR, HER2, HER3, and HER4, has been tested in a phase II trial with esophagogastric cancer who progressed to trastuzumab, showing an ORR of $10 \% .{ }^{34} \mathrm{An}$ ongoing phase II study is currently evaluating afatinib combined with paclitaxel in previously treated patients with anti-HER2 therapy (NCT02501603; NCT01522768).

Interesting results have recently been achieved with trastuzumab deruxtecan (DS-8201), an anti-HER2 monoclonal antibody conjugate with a cleavable tetrapeptidebased linker and a cytotoxic topoisomerase I inhibitor. ${ }^{35}$ The most relevant feature is the "bystander killing effect" consisting in the internalization of trastuzumab deruxtecan mediated by HER2-positive cells, the release of deruxtecan into the cytoplasm and the subsequent transfer into adjacent HER2-negative cells. Thereby, trastuzumab deruxtecan could overcome the heterogeneity of HER2 expression in GC. DS-8201 has a higher drug-toantibody ratio compared to T-DM1 (approximately 8 vs 3-4) associated with higher cytotoxic payload on HER2 positive cancer cells. Furthermore, its high cytotoxic effect could also be extended due to high membrane permeability. In PDX model, trastuzumab deruxtecan demonstrated high activity in resistant tumors, probably due to higher p-glycoprotein mediated efflux for T-DM1.

Based on encouraging results in breast cancer and the Phase I trial in GC patients, the phase II trial, DESTINYGastric01, randomized GC patients treated with two previous lines to receive trastuzumab deruxtecan versus physician choice's (ORR 51.3\% in the experimental arm and 14.3\% in the control arm). ${ }^{36,37}$ Accordingly, FDA has recently approved trastuzumab deruxtecan for the treatment of HER2positive GC patients who have received a prior trastuzumabbased therapy ${ }^{37}$ Table 1.

More recently, among anti-HER2 monoclonal antibody under development, margetuximab has emerged as a promising agent in treating HER2-positive GC. It is a next-generation $\mathrm{Fc}$-modified chimeric antibody with ADCC activity. ${ }^{38}$ In a phase Ib/II trial in advanced HER2positive GC patients, regardless PD-L1 expression, margetuximab plus pembrolizumab has been evaluated in second-line therapy. Preliminary results showed an acceptable profile of toxicity and an objective responses rate of $18.4 \%$, supporting the synergistic activity between anti-HER2 treatments and immune modulation treatments. $^{39}$ The ongoing phase II/III MAHOGANY trial randomized HER2-positive GC to receive margetuximab combined with an anti-PD-1 monoclonal antibody, INCMGA00012 (Cohort A) and margetuximab combined with INCMGA00012 or MGD013 (anti-PD-1/anti-LAG-3) and chemotherapy compared to trastuzumab combined with chemotherapy (Cohort B) NCT04082364.

\section{Immune Checkpoints Inhibitors for Selected Profiles}

In the last few years, impressive improvements have been achieved in many different cancers thanks to novel immunotherapy strategies. ${ }^{40-42}$ Recently, its role has been investigated also in gastrointestinal cancers, including GC. Immune system plays a key role in cancer growth control. Cancer cells, through immune checkpoint pathways the recruitment of inhibitory and co-inhibitory molecules, escape immune response. In a first phase of cancer development, the release of tumor antigens and danger molecules activates the innate and adaptive immune system. However, after initial killing phase of high immunogenicity clones, several clones variants could develop leading the escape of immune system control. In particular, cancer subverts immune cells by activating inhibitors pathways, such as CTLA4 and PD1. CTLA4 binding CD28, produces inhibitory signals. PD-1 binding PD-L1/2 stimulates apoptosis in T-lymphocytes. Immune checkpoint inhibitors, by avoiding binding with their ligands, can reestablish immune activity against cancer cells. Although interesting data are available in molecularly selected GC patients, the clinical impact of immune checkpoints inhibitors for the overall population is still under investigation in several ongoing clinical trials. ${ }^{43}$ Developing strong immunotherapeutic strategies in GC is made more complicated by the broad heterogeneity of different tumor subtypes that involves the lack of potential predictive biomarkers. ${ }^{7}$

Pembrolizumab is an IgG4 humanized antibody targeting programmed cell death protein-1 (PD-1) receptor of lymphocytes. Nowadays, it is the only FDA-approved immunotherapy for heavily pre-treated PD-L1 CPS positive and microsatellite instability-high (MSI-H) GC 
Table I Clinical Trials Supporting the Use of Molecular Matched Therapy in GC

\begin{tabular}{|c|c|c|c|c|c|c|c|}
\hline Trial & Phase & Experimental Arm & Control Arm & $\begin{array}{l}\text { Line of } \\
\text { Therapy }\end{array}$ & $\begin{array}{l}\text { HR } \\
\text { for OS }\end{array}$ & $P$ value & $95 \% \mathrm{Cl}$ \\
\hline \multicolumn{8}{|l|}{ Anti-HER2 agents } \\
\hline TOGA & III & $\begin{array}{l}\text { Trastuzumab plus Cisplatin+5-FU/ } \\
\text { capecitabine }\end{array}$ & $\begin{array}{l}\text { Cisplatin+5-FU/ } \\
\text { capecitabine }\end{array}$ & First & 0.74 & 0.0046 & $0.60-0.90$ \\
\hline TRIO-0I3/LOGIC & III & $\begin{array}{l}\text { Oxaliplatin, capecitabine plus } \\
\text { Lapatinib }\end{array}$ & $\begin{array}{l}\text { Oxaliplatin, } \\
\text { capecitabine }\end{array}$ & First & 0.91 & 0.35 & $0.73-1.12$ \\
\hline TYTAN & III & Paclitaxel plus Lapatinib & Lapatinib & Second & 0.84 & 0.10 & $0.64-1.11$ \\
\hline$J A C O B$ & III & $\begin{array}{l}\text { Cisplatin+5-FU/ capecitabine / } \\
\text { Trastuzumab plus Pertuzumab }\end{array}$ & $\begin{array}{l}\text { Cisplatin+5-FU/ } \\
\text { capecitabine / } \\
\text { Trastuzumab }\end{array}$ & First & 0.84 & 0.057 & $0.7 I-1.00$ \\
\hline GATSBY & $\| / I I I$ & TDM-I & Taxane & Second & 1.15 & 0.85 & $0.87-|.5|$ \\
\hline $\begin{array}{l}\text { DESTINY- } \\
\text { Gastric0I }\end{array}$ & II & Trastuzumab Deruxtecan & Paclitaxel or Irinotecan & Third & 0.59 & 0.01 & $0.39-0.88$ \\
\hline \multicolumn{8}{|c|}{ Immune checkpoint inhibitors } \\
\hline KEYNOTE-059 & ॥ & Pembrolizumab & - & Third or later & & & \\
\hline KEYNOTE-06I & III & Pembrolizumab & Paclitaxel & Second & 0.82 & 0.04 & $0.66-1.03$ \\
\hline KEYNOTE-062 & III & $\begin{array}{l}\text { Pembrolizumab or } \\
\text { Pembrolizumab plus CT }\end{array}$ & $\mathrm{CT}$ plus placebo & First & $\begin{array}{l}0.91 ; \\
0.85\end{array}$ & & $\begin{array}{l}0.69-1.18 \\
0.70-1.03\end{array}$ \\
\hline ATTRACTION-2 & III & Nivolumab & Placebo & Third or later & 0.63 & 0.0001 & $0.5 \mathrm{I}-0.78$ \\
\hline CheckMate-032 & $\mathrm{I} / \mathrm{II}$ & $\begin{array}{l}\text { Ipilimumab plus Nivolumab or } \\
\text { Nivolumab }\end{array}$ & $\begin{array}{l}\text { Nivolumab plus } \\
\text { Ipilimumab }\end{array}$ & Third & & & \\
\hline CHECKMATE-649 & III & $\begin{array}{l}\text { Nivolumab or Ipilimumab + } \\
\text { Nivolumab }\end{array}$ & CapeOX e FOLFOX & First & 0.80 & 0.0001 & $0.59-0.78$ \\
\hline \multicolumn{8}{|c|}{ Anti-angiogenic treatment } \\
\hline AVAGAST & III & $\begin{array}{l}\text { Bevacizumab, Cisplatin and } \\
\text { Capecitabine }\end{array}$ & $\begin{array}{l}\text { Cisplatin and } \\
\text { Capecitabine }\end{array}$ & First & 0.87 & 0.10 & $\begin{array}{l}0.73 \text { to } \\
1.03\end{array}$ \\
\hline RAINBOW & III & Ramucirumab plus Paclitaxel & Paclitaxel & Second & 0.80 & 0.01 & $0.67-0.96$ \\
\hline RAINFALL & III & $\begin{array}{l}\text { Cisplatin+5-FU/ capecitabine plus } \\
\text { Ramucirumab }\end{array}$ & $\begin{array}{l}\text { Cisplatin+5-FU/ } \\
\text { capecitabine }\end{array}$ & First & 0.96 & 0.67 & $0.80-1 \cdot 15$ \\
\hline \multicolumn{8}{|c|}{ Drugs targeting tight-junction Proteins } \\
\hline FAST & ॥ & Zolbetuximab plus EOX & EOX & First & 0.56 & 0.001 & $0.40-0.79$ \\
\hline \multicolumn{8}{|l|}{ PARP inhibitors } \\
\hline GOLD & III & Olaparib plus paclitaxel & Paclitaxel plus placebo & Second & 0.79 & 0.026 & $0.64-1.00$ \\
\hline
\end{tabular}

patients $^{44}$ on the basis of single-arm multicohort multicenter trials (KEYNOTE-016, -164, -012, -028 , and $-158) .{ }^{45}$ The Phase $1 \mathrm{~b}$ multicohort KEYNOTE-012 trial showed that pembrolizumab obtained a $22 \%$ of disease partial response in PD-L1 positive chemo-refractory patients. ${ }^{46}$ In the Phase 2 trial KEYNOTE-059 a durable overall response rate (ORR) of $22.7 \%$ was observed in PDL-1 positive patients with a median response duration of 8.1 months, compared with an ORR of $16.4 \%$ in the overall study population. ${ }^{44}$ 
In the KEYNOTE-061 trial, patients progressing on first-line therapy were randomized to receive either pembrolizumab for up 2 years or standard second-line therapy. Overall, pembrolizumab did not demonstrate any benefit in the primary endpoints of OS and PFS in patients with PDL1 positive tumors. However, the post-hoc analysis suggested a clear benefit for pembrolizumab in both tumors with PDL-1 expression $>10 \%$ with median OS of 10.4 month (vs 8 months) and a RR of $24.5 \%$ (vs 9.1) and in patients with MSI-H, irrespective of the PDL-1 value (HR 0.42 with median OS not reached). ${ }^{47}$

Notably, in the ATTRACTION-2 Phase 3 trial, nivolumab, an IgG4 human monoclonal antibody anti-PD-1, was evaluated as monotherapy versus placebo in heavily pretreated GC patients. Nivolumab obtained significant survival benefit with median OS of 5.26 versus 4.14 months (HR 0.62, 95\% CI 0.51-0.78; p<0.0001) and 12-month overall survival rate of $26.2 \%$ versus $10.9 \%$, regardless of PDL-1 status. ${ }^{48}$

The phase $1 / 2$ CheckMate-032 trial showed a $24 \%$ ORR with the combination of nivolumab and ipilimumab, an IgG1 human monoclonal antibody anti-cytotoxic T-lymphocyte antigen-4 (CTLA-4), in GC pretreated patients, regardless of PDL-1 and MSI status. Anyway, ORR seemed higher in patients with PD-L1 positive or MSI-H subtypes. ${ }^{49}$

An open-label randomized phase 3 trial (CheckMate649) investigating the role of upfront ipilimumab plus nivolumab in comparison to nivolumab plus chemotherapy and to standard chemotherapy alone was conducted in first-line advanced GC patients, regardless of PD-L1. First-line nivolumab in combination with chemotherapy showed a statistically significant improvement in OS and PFS versus chemotherapy alone in HER 2 negative GC patients with tumors expressed PDL-1 CPS $>5$ (OS HR $0.7198 .4 \%$ CI $0.59-0.86 \mathrm{p}<0.0001$ ) and (PFS: HR 0.68 $98 \%$ CI $0.56-0.81 ; p<0.0001)$. Significant benefit in terms of OS was also observed in patients with CPS $>11$ (HR $0.77,99.3 \%$ CI $0.64-0.92, \mathrm{p}<0.0001)$ and in the overall population (HR $0.80,99.3 \%$ CI $0.68-0.94, \mathrm{p}<0.0001$ ). No unexpected toxicities were registered.

The phase 3 randomized trial, KEYNOTE-062, of pembrolizumab \pm chemotherapy versus chemotherapy alone in first-line therapy enrolled 763 patients with HER2-negative advanced GC patients. Pembrolizumab monotherapy resulted in non-inferior OS compared with standard chemotherapy: median OS was 10.6 months for those receiving pembrolizumab compared with 11.1 months for those who received chemotherapy (HR 0.91; 99.2\% CI $0.69-1.18 ; \mathrm{p}<0.0001)$ with less toxicities. In patients with a PD-L1 combined positive score (CPS) of 10 or more and MSI-H, a clinically significant improvement of survival was found (17.4 months for those receiving pembrolizumab compared with 10.8 months for those receiving chemotherapy [HR $0.69 ; 95 \%$ CI $0.49-0.97$; $\mathrm{p}<0.0001]) .{ }^{50}$

Moreover, in the KEYNOTE-062 trial the combination of pembrolizumab with standard chemotherapy failed to improve both OS and PFS of patients with PD-L1 positive (with CPS 1 or higher) GC (HR 0.85; 95\% 0.70-1.03; $\mathrm{p}=$ $0.046){ }^{50}$

The ATTRACTION-4 trial, designed similarly to CheckMate-649, was conducted in Asian GC patients. Nivolumab plus chemotherapy improved the co-primary endpoint PFS, supporting results obtained in CheckMate649. However, OS was not improved, likely due to inclusion of all-comers or more subsequent lines treatment proposed to Asian patients compared to Western subjects.

Based on all these results, pembrolizumab was approved by FDA for PD-L1 positive chemo-refractory GC patients while nivolumab only in Japan for all chemorefractory patients.

Toripalimab, anIgG4 monoclonal antibody anti-PD1, was evaluated in a Chinese multi-center phase $1 b / 2$ trial [NCT02915432] as monotherapy or added with standard chemotherapy in GC patients who progressed to standard treatment. Patients were divided into 2 groups depending on their TMB status. Patients in the TMB-high group showed a significant OS advantage than patients in the TMB-low group (14.6 versus 4.0 months, HR $0.48, \mathrm{p}=$ $0.038)^{51}$ Table 1.

Other phase $2 / 3$ clinical trials evaluating several immune checkpoint inhibitors are ongoing, including atezolizumab (anti-PDL1) [NCT04661150], avelumab (anti-PDL1), ${ }^{52}$ durvalumab (anti-PDL1), ${ }^{53}$ stintilimab (anti-PD1) [NCT03 745170], tislelizumab (anti-PD1) [NCT03469557], retifanlimab (anti-PD1) [NCT04082364], camrelizumab (anti-PD1) [NCT03472365], tremelimumab (an anti-CTLA 4) ${ }^{15}$ relatlimab (an anti-LAG3) [NCT01968109] and tebotelimab (an anti-CTLA-4 and antiPD1 bispecific DART protein) [NCT04082364].

Furthermore, different strategies are emerging to overcome primary and secondary resistance to checkpoint inhibition. In particular, tumor macrophages-associated (TAMs) were found to play a central role in tumor promotion and immunoediting in several cancer types. ${ }^{54} \mathrm{~A}$ direct 
inhibition of TAM through targeting (CSF-1)/CSF-1 receptor (CSF-1R) is under development in early phase clinical trials. First phase 1 data showed promising activity of emactuzumab (RG7155; Celleron Therapeutics), a monoclonal IgG1 antibody against CSF-1R and further clinical investigation is ongoing. ${ }^{55-57}$

For resectable GC and gastroesophageal patients, two randomized phase 3 clinical trials of perioperative chemotherapy with immunotherapy are ongoing (KEYNOTE-585, NCT03221426 and DANTE trial, NCT034212889). PFS is the primary endpoint of the first trial evaluating perioperative pembrolizumab plus cisplatin/5FU versus cisplatin/5FU while pathological CR is the primary endpoint of the DANTE trial evaluating the combination of atezolizumab with FLOT versus FLOT in the same perioperative setting.

Overall, clinical data suggest a significant clinical benefit of checkpoints inhibitors approaches in particular in MSI-H advanced GC, accounting for 5\% of advanced and $10-20 \%$ of localized disease. For patients with MSI-H tumors, the benefit of pembrolizumab seems consistent regardless of the line of therapy in which it was received. ${ }^{58}$

Role of combination of different checkpoints inhibitors or with standard chemotherapy is still not defined and further clinical investigation is required.

\section{Angiogenesis Targeting Drugs}

Angiogenesis, especially vascular endothelial growth factor (VEGF) plays a key role in cancer growth control and metastatic spreading in several cancer types, including GC. Thereby, its targeting represents a significant therapeutic approach. ${ }^{59}$

Despite milestone advances in many other diseases, bevacizumab - an anti-VEGF humanized monoclonal antibody has yielded disappointing results in terms of OS compared to standard chemotherapy in the first-line setting for advanced GC patients in two phase III trials. ${ }^{60,61}$ Of note, subgroup analyses revealed a different response to anti-angiogenic treatment in different genetic populations (no clinical benefit for Chinese patients instead of a significant survival extension for American patients).

Furthermore, anti-angiogenic treatment with bevacizumab among resectable patients did not improve OS compared to chemotherapy alone in the preoperative setting, even if improving PFS and rates of complete surgical resection. ${ }^{62}$ Moreover, Cunningham et al reported higher incidence of anastomotic dehiscence for patients treated with bevacizumab. ${ }^{63}$ Based on these results, bevacizumab is not approved for the treatment of GC patients.

Ramucirumab, a human IgG1 monoclonal antibody that binds to VEGF receptor (VEGFR)-2 on the endothelial cells in blood vessels and inhibit VEGF ligand binding and receptor signaling, showed a significant benefit in terms of survival outcomes of pretreated patients with advanced GC in two phase III trials. ${ }^{4,6,64}$ In both REGARD (ramucirumab $8 \mathrm{mg} / \mathrm{kg}$ vs placebo every 2 weeks) and RAINBOW (ramucirumab $8 \mathrm{mg} / \mathrm{kg}$ or placebo every 2 weeks in combination with weekly paclitaxel $80 \mathrm{mg} / \mathrm{m} 2$ ) trials, clinical outcomes of patients treated with ramucirumab were significantly improved with an acceptable tolerability profile. In particular, in REGARD trial median OS was 5.2 months for the ramucirumab group vs 3.8 months in the placebo group (HR 0.77, 95\% CI 0.60-0.99; p=0.047). In RAINBOW, median OS was 9.8 months for combined treatment vs 7.4 months in the placebo arm (HR 0.80; 95\% CI 0.67-0.96; $\mathrm{p}=0.017$ ). Based on these results, ramucirumab is currently approved alone or in combination with paclitaxel for the treatment of advanced GC patients who have progressed to first-line platinum-based therapy. ${ }^{64}$ Notably, the randomized first-line phase III trial RAINFALL failed to show any improvement from the combination of ramucirumab with standard chemotherapy (HR 0.96, 95\% 0.80-1.15, p=0.675, median OS 11.2 vs 10.7 months in the placebo group). ${ }^{65}$ Interestingly, the RAMSES/FLOT7 phase II trial are currently testing ramucirumab in combination with perioperative chemotherapy in addition with FLOT- based regimen (fluorouracil/oxaliplatin/docetaxel) [NCT02661971].

Other antiangiogenic strategies have been tested for GC patients. Among them, apatinib, in a novel smallmolecule inhibitor of VEGFR-2 by blocking its intracellular domain. Compared to placebo, apatinib showed to prolong OS (6.5 vs 4.7 months; $\mathrm{HR}=0.71$; 95\% CI, $0.54-$ $0.94 ; \mathrm{p}=0.015$ ) and PFS (2.6 vs 1.8 months; $\mathrm{HR}=0.44$; $\mathrm{p}<0.001$ ) in GC patients progressed to first-line therapy in a phase III trial. The authors reported low rates of severe toxicity with $21 \%$ of patients requiring a dose reduction. ${ }^{66}$

Anti-angiogenic agents can influence the immunosuppressive tumour microenvironment which rouses cancer resistance to anti-PD-1/PD-L1 treatment strategies. Camrelizumab (SHR-1210) (INN) is an anti-PD-1 immune checkpoint inhibitor that showed promising antitumor activity in early phase clinical trials. In a multicenter, open-label, phase II trial, which assessed camrelizumab in combination with chemotherapy followed by camrelizumab plus apatinib as a first-line therapy for advanced 
GC, the ORR was $58.3 \%$ (95\% CI, 43.2-72.4) with mOS of 14.9 months (95\% CI, 13.0-18.6), and mPFS of 6.8 months (95\% CI, 5.6-9.5) with manageable toxicity [NCT04067986].

Lastly, regorafenib, a multi kinase inhibitor blocking VEGFR-1 and 2, demonstrated to prolong PFS in the phase II INTEGRATE trial ${ }^{67}$ in which 147 patients were randomized 2:1 to receive regorafenib (160 $\mathrm{mg}$ daily, days 1-21 on a 28 -day cycle) or placebo after disease progression on 1 or 2 lines of chemotherapy. The treatment was generally well-tolerated. ${ }^{67}$ In a systematic review among 16 trials of TKIs in GC only apatinib and regorafenib showed positive results in terms of clinical benefit compared to standard treatment. ${ }^{68}$ These overall disappointing results could be linked to the rapid development of escape pathways in GC that overcome anti-angiogenic inhibition. Identifying potential predictive biomarkers will be crucial for developing new selected therapeutic strategies.

\section{Novel Potential Signaling Pathways Claudin 18.2}

In recent years, the tight junction molecule Claudin 18 isoform 2 has been evaluated as a potential therapeutic target in GC patients. ${ }^{69}$ Claudins are proteins controlling the interchange of molecules among cells. They are expressed on stomach, pancreas and lung, whilst Claudin 18.2 is distributed only in gastric tissues, and it is reported both in HER2 positive and in HER2 negative cancers. During carcinogenesis, tight junctions expose Claudin 18.2 epitopes expressed on cancer cells. ${ }^{70}$

Zolbetuximab, the first agent anti-Claudin 18.2 developed, is a IgG1 monoclonal antibody acting through inhibition of cell growth triggering ADCC and complement-dependent cytotoxicity (CDC).$^{71}$

A phase II trial, MONO 2013, showed an interesting clinical benefit in refractory GC patients with Claudin 18.2-positive tumors defined as an IHC score $\geq 2+$ in more than $50 \%$ of cancer cells treated with zolbetuximab as single agent. ${ }^{72}$ Notably, among 40 patients who received claudiximab (or called zolbetuximab), the ORR was $10 \%$, and the disease control rate was $30 \%$. Henceforth, the randomized phase II FAST trial demonstrated that zolbetuximab plus EOX confers a survival improvement over EOX alone (mOS: 13.3 vs 8.4 months, respectively; HR 0.56 ; $95 \%$ CI $0.40,0.79, \mathrm{p}<0.001$; mPFS 7.9 vs 4.8 months; HR 0.47 ; $=0.0001)$ along with a better ORR ( $39 \%$ vs $25 \%$; $\mathrm{P}=0.022)$ in $\mathrm{GC}$ patients with Claudin 18.2-positive (Table 1). Therefore, several randomized studies, such as GLOW, ILUSTRO and SPOTLIGHT are currently investigating the role of zolbetuximab in improving clinical outcomes in advanced GC combined with a platinum-based therapy. ${ }^{73}$ Noteworthy, a subgroup analysis of FAST trial demonstrated that Claudin 18.2 expression in $\geq 70 \%$ of cancer cells was associated with better OS (HR 0.44), leading to further patient enrichment $(\geq$ $75 \%$ of tumor cells) in the ongoing phase III SPOTLIGHT trial, evaluating zolbetuximab in association with FOLFOX in first-line setting. ${ }^{74}$

\section{FGFR Pathway}

Several molecular targeting agents did not show significant improvement in clinical studies, probably due to inappropriate patient selection and molecular stratification.

Interestingly, Pearson et al reported high response rate in advanced GC expressing high level of FGF2 treated with FGFR inhibitor AZD4547. ${ }^{75}$ FGFR pathway consists of five kinase receptors that are activated by FGF inducing cell survival and proliferation. ${ }^{76,77}$

Notably, the FGFR2b variant is overexpressed in approximately $2-30 \%$ of GC and, in preclinical studies, it is associated with an FGFR2 gene amplification. ${ }^{10,77,78} \mathrm{GC}$ is associated with worse prognosis in patients with FGFR2b overexpression or FGFR2 amplification. Of note, this latter often occur in CIN and GS molecular subtypes. ${ }^{79}$

Moreover, many studies provided increasing data of FGFR pathway in acquiring resistance to trastuzumabbased treatment, particularly mediated by overexpression of FGFR3 and FGF9 that are associated with phosphorylated AKT, in addition to losing HER2 expression. ${ }^{33}$

The phase II SHINE trial randomized advanced GC patients presenting FGFR2 polysomy or gene amplification to receive AZD4547 or paclitaxel. AZD4547 was not associated with PFS improvement over standard chemotherapy. ${ }^{80}$ A biomarker analysis suggested a correlation between the lack of efficacy and the intratumor heterogeneity for FGFR2 gene expression. ${ }^{80}$

Bemarituzumab is an afucosylated humanized monoclonal antibody that inhibits FGFR2b on cancer cells, thus blocking growth factor signaling. In the FIGHT trial, a double blind study enrolling patients with advanced GC that were treated either with bemarituzumab added to standard chemotherapy or chemotherapy alone, the investigators reported for FGFR positive patients treated with the experimental drug a 2-month improvement in terms of median PFS (9.5 vs 7.4 months) and an higher response rate (47\% 
vs $33 \%) .{ }^{81}$ Notably, more corneal adverse events, stomatitis and nausea were linked to bemarituzumab.

\section{DNA Damage Response Pathway}

Alteration of the DNA damage response characterized chromosomal instability (CIN) and promote carcinogenesis. In particular, ataxia-telangiectasia mutated (ATM) proteins plays a key role in the DNA double strand breaks. Thereby, ATM deficiency, harbored up to $22 \%$ of advanced GC, is associated with sensitivity to poly(ADPribose) polymerase (PARP) inhibitors. ${ }^{16,82}$

The phase III GOLD trial failed to demonstrate significant benefit from adding olaparib to taxane, both in the whole unselected subgroup and ATM-negative population (mOS 8.8 months in the olaparib group vs 6.9 months with placebo; HR 0.79, 95\% CI 0.64-1.00; $\mathrm{P}=0.026)^{83}$ (Table 1).

Ongoing phase III studies are testing maintenance treatments in advanced GC patients after first-line platinum-based treatment.

\section{EGFR Inhibitors}

Despite the use of several Epidermal Growth Factor Receptor (EGFR) inhibitors having been approved in colorectal malignancies, head and neck tumors and specific subgroup of EGFR-mutated advanced non-small cell lung cancers, their use in gastric cancer remains controversial. A number of phase III RCTs evaluating the use of cetuximab, panitumumab, or gefitinib combined to the standard of care in molecularly unselected gastric cancer patients reported negative results, since this combination failed to improve the outcome of patients with locally advanced or metastatic disease. For example, no benefit was found adding panitumumab to FOLFOX ${ }^{84}$ or EOX. ${ }^{85}$ Also, no survival increase was reported adding cetuximab to capecitabine and cisplatin combination. ${ }^{86}$ Result of the use of EGFR-inhibitors in molecularly selected tumors are similarly disappointing, and EGFR inhibitors are not licensed for clinical use to treat advanced gastric cancer.

Nevertheless, novel translational research and experimental preclinical data showed a positive correlation between cetuximab response and high EGFR expression/ amplification, which occur in $3-4 \%$ of all cases, and let investigator rethink on how to optimize the treatment strategy. In fact, a recent study conducted in PDX xenopatien models has confirmed that EGFR inhibitors may be active in EGFR-addicted gastric adenocarcinoma with EGFR copynumber gain $^{87}$ and may serve as springboard for future research to identify potentially actionable targets and resistance mechanisms.

\section{Conclusions}

Despite chemotherapy having long been the gold standard of treatment for advanced GC, we are now moving towards a new era of developing targeted agents for genes and signaling pathways. However, a widespread approach towards personalized oncology is still far away. Further studies embracing the crosstalk among tumor microenvironment, host characteristics and cancer molecular make-up are necessary to better identify those patients that could benefit from monoclonal antibodies, in order to optimize treatment strategies according to novel molecular pathways.

Chemotherapy combined with trastuzumab is the current standard first-line setting for HER2 positive advanced GC and recent more active anti-HER2 agents are leading to improved survival in this subgroup. Anti-VEGF inhibitors, in particular ramucirumab, has been approved for the use in the second-line treatment. Immune checkpoint inhibitors represent a promising option in MSI high, EBV positive or tumors with strong PD-L1 expression. In this moving landscape, the possibility to specifically target HER2, Claudine 8.2, FGFR, and many other oncogenic alterations will hopefully improve survival outcomes of advanced GC patients.

\section{Disclosure}

The authors report no conflicts of interest in this work.

\section{References}

1. Ferlay J, Soerjomataram I, Dikshit R, et al. Cancer incidence and mortality worldwide: sources, methods and major patterns in GLOBOCAN 2012. Int $J$ Cancer. 2015;136:E359-E386. doi:10.10 02/ijc. 29210

2. Koizumi W, Narahara H, Hara T, et al. S-1 plus cisplatin versus S-1 alone for first-line treatment of advanced gastric cancer (SPIRITS trial): a phase III trial. Lancet Oncol. 2008;9:215-221. doi:10.1016/ S1470-2045(08)70035-4

3. Cunningham D, Starling N, Rao S, et al. Capecitabine and oxaliplatin for advanced esophagogastric cancer. $N$ Engl J Med. 2008; 358:36-46. doi:10.1056/NEJMoa073149

4. Wilke H, Muro K, Van Cutsem E, et al. Ramucirumab plus paclitaxel versus placebo plus paclitaxel in patients with previously treated advanced gastric or gastro-oesophageal junction adenocarcinoma (RAINBOW): a double-blind, randomised phase 3 trial. Lancet Oncol. 2014;15:1224-1235. doi:10.1016/S1470-2045(14)70420-6

5. Janowitz T, Thuss-Patience P, Marshall A, et al. Chemotherapy vs supportive care alone for relapsed gastric, gastroesophageal junction, and oesophageal adenocarcinoma: a meta-analysis of patient-level data. Br J Cancer. 2016;114:381-387. doi:10.1038/bjc.2015.452

6. Fuchs CS, Tomasek J, Yong CJ, et al. Ramucirumab monotherapy for previously treated advanced gastric or gastro-oesophageal junction adenocarcinoma (REGARD): an international, randomised, multicentre, placebo-controlled, phase 3 trial. Lancet. 2014;383:31-39. doi:10.1016/S0140-6736(13)61719-5

7. Garattini SK, Basile D, Cattaneo M, et al. Molecular classifications of gastric cancers: novel insights and possible future applications. World J Gastrointest Oncol. 2017;9:194-208. doi:10.4251/wjgo.v9.i5.194 
8. Cunningham D, Allum WH, Stenning SP, et al. Perioperative chemotherapy versus surgery alone for resectable gastroesophageal cancer. N Engl J Med. 2006;355:11-20. doi:10.1056/NEJMoa055531

9. Al-Batran S-E, Homann N, Pauligk C, et al. Perioperative chemotherapy with fluorouracil plus leucovorin, oxaliplatin, and docetaxel versus fluorouracil or capecitabine plus cisplatin and epirubicin for locally advanced, resectable gastric or gastro-oesophageal junction adenocarcinoma (FLOT4): a randomised, phase 2/3 trial. Lancet. 2019;393:1948-1957. doi:10.1016/S0140-6736(18)32557-1

10. Cancer Genome Atlas Research Network. Comprehensive molecular characterization of gastric adenocarcinoma. Nature. 2014;513 (7517):202-209. doi:10.1038/nature13480.

11. Cristescu R, Lee J, Nebozhyn M, et al. Molecular analysis of gastric cancer identifies subtypes associated with distinct clinical outcomes. Nat Med. 2015;21:449-456. doi:10.1038/nm.3850

12. Kim JW, Nam KH, Ahn S-H, et al. Prognostic implications of immunosuppressive protein expression in tumors as well as immune cell infiltration within the tumor microenvironment in gastric cancer. Gastric Cancer. 2016;19:42-52. doi:10.1007/s10120-014-0440-5

13. Ishigami S, Natsugoe S, Tokuda K, et al. Prognostic value of intratumoral natural killer cells in gastric carcinoma. Cancer. 2000;88:577-583. doi:10.1002/(SICI)1097-0142(20000201)88:3<57 7::AID-CNCR13>3.0.CO;2-V

14. Grogg KL, Lohse CM, Pankratz VS, Halling KC, Smyrk TC. Lymphocyte-rich gastric cancer: associations with Epstein-Barr virus, microsatellite instability, histology, and survival. Mod Pathol. 2003;16:641-651. doi:10.1097/01.MP.0000076980.73826.C0

15. Lazăr DC, Avram MF, Romoșan I, Cornianu M, Tăban S, Goldiș A. Prognostic significance of tumor immune microenvironment and immunotherapy: novel insights and future perspectives in gastric cancer. World J Gastroenterol. 2018;24:3583-3616. doi:10.3748/ wjg.v24.i32.3583

16. Kim ST, Banks KC, Pectasides E, et al. Impact of genomic alterations on lapatinib treatment outcome and cell-free genomic landscape during HER2 therapy in HER2+ gastric cancer patients. Ann Oncol. 2018;29:1037-1048. doi:10.1093/annonc/mdy034

17. Kato S, Okamura R, Baumgartner JM, et al. Analysis of circulating tumor DNA and clinical correlates in patients with esophageal, gastroesophageal junction, and gastric adenocarcinoma. Clin Cancer Res. 2018;24:6248-6256. doi:10.1158/1078-0432.CCR-18-1128

18. Citri A, Yarden Y. EGF-ERBB signalling: towards the systems level. Nat Rev Mol Cell Biol. 2006;7:505-516. doi:10.1038/nrm1962

19. Nahta R, Yuan LXH, Zhang B, Kobayashi R, Esteva FJ. Insulin-like growth factor-I receptor/human epidermal growth factor receptor 2 heterodimerization contributes to trastuzumab resistance of breast cancer cells. Cancer Res. 2005;65:11118-11128. doi:10.1158/00085472.CAN-04-3841

20. Brufsky A. Trastuzumab-based therapy for patients with HER2-positive breast cancer: from early scientific development to foundation of care. Am J Clin Oncol. 2010;33:186-195. doi:10.1097/ COC.0b013e318191bfb0

21. Bang Y-J, Van Cutsem E, Feyereislova A, et al. Trastuzumab in combination with chemotherapy versus chemotherapy alone for treatment of HER2-positive advanced gastric or gastro-oesophageal junction cancer (ToGA): a phase 3, open-label, randomised controlled trial. Lancet. 2010;376:687-697. doi:10.1016/S0140-6736(10)61121-X

22. Hecht JR, Bang YJ, Qin SK, et al. Lapatinib in combination with capecitabine plus oxaliplatin in human epidermal growth factor receptor 2-positive advanced or metastatic gastric, esophageal, or gastroesophageal adenocarcinoma: TRIO-013/LOGiC-a randomized phase III trial. J Clin Oncol. 2016;34(5):443-451. doi:10.1200/JCO.2015.62.6598

23. Satoh T, Xu R-H, Chung HC, et al. Lapatinib plus paclitaxel versus paclitaxel alone in the second-line treatment of HER2-amplified advanced gastric cancer in asian populations: TyTAN - a randomized, phase III study. J Clin Oncol. 2014;32:2039-2049. doi:10.1200/JCO.2013.53.6136
24. Tabernero J, Hoff PM, Shen L, et al. Pertuzumab plus trastuzumab and chemotherapy for HER2-positive metastatic gastric or gastro-oesophageal junction cancer (JACOB): final analysis of a double-blind, randomised, placebo-controlled phase 3 study. Lancet Oncol. 2018;19:1372-1384. doi:10.1016/S1470-2045(18)30481-9

25. Thuss-Patience PC, Shah MA, Ohtsu A, et al. Trastuzumab emtansine versus taxane use for previously treated HER2-positive locally advanced or metastatic gastric or gastro-oesophageal junction adenocarcinoma (GATSBY): an international randomised, open-label, adaptive, phase 2/3 study. Lancet Oncol. 2017;18:640-653. doi:10.1016/S1470-2045(17)30111-0

26. Li Q, Jiang H, Li H, et al. Efficacy of trastuzumab beyond progression in HER2 positive advanced gastric cancer: a multicenter prospective observational cohort study. Oncotarget. 2016;7:50 656-50665. doi:10.18632/oncotarget.10456

27. Yang J, Luo H, Li Y, et al. Intratumoral heterogeneity determines discordant results of diagnostic tests for human epidermal growth factor receptor (HER) 2 in gastric cancer specimens. Cell Biochem Biophys. 2012;62:221-228. doi:10.1007/s12013-011-9286-1

28. Kaito A, Kuwata T, Tokunaga M, et al. HER2 heterogeneity is a poor prognosticator for HER2-positive gastric cancer. World J Clin Cases. 2019;7:1964-1977. doi:10.12998/wjcc.v7.i15.1964

29. Bartley AN, Washington MK, Colasacco C, et al. HER2 testing and clinical decision making in gastroesophageal adenocarcinoma: guideline from the College of American Pathologists, American Society for Clinical Pathology, and the American Society of Clinical Oncology. J Clin Oncol. 2017;35:446-464. doi:10.1200/JCO.2016.69.4836

30. Pectasides E, Stachler MD, Derks S, et al. Genomic heterogeneity as a barrier to precision medicine in gastroesophageal adenocarcinoma. Cancer Discov. 2018;8:37-48. doi:10.1158/2159-8290.CD-17-0395

31. Wang H-B, Liao X-F, Zhang J. Clinicopathological factors associated with HER2-positive gastric cancer: a meta-analysis. Medicine (Baltimore). 2017;96:e8437. doi:10.1097/MD.0000000000008437

32. Janjigian YY, Sanchez-Vega F, Jonsson P, et al. Genetic predictors of response to systemic therapy in esophagogastric cancer. Cancer Discov. 2018;8:49-58. doi:10.1158/2159-8290.CD-17-0787

33. Piro G, Carbone C, Cataldo I, et al. An FGFR3 autocrine loop sustains acquired resistance to trastuzumab in gastric cancer patients. Clin Cancer Res. 2016;22:6164-6175. doi:10.1158/10780432.CCR-16-0178

34. Sanchez-Vega F, Hechtman JF, Castel P, et al. EGFR and MET amplifications determine response to HER2 inhibition in ERBB2-amplified esophagogastric cancer. Cancer Discov. 2019;9:199-209. doi:10.1158/2159-8290.CD-18-0598

35. Ogitani Y, Aida T, Hagihara K, et al. DS-8201a, a novel HER2-targeting ADC with a novel DNA topoisomerase I inhibitor, demonstrates a promising antitumor efficacy with differentiation from T-DM1. Clin Cancer Res. 2016;22:5097-5108. doi:10.1158/ 1078-0432.CCR-15-2822

36. Takegawa N, Tsurutani J, Kawakami H, et al. [Fam-] trastuzumab deruxtecan, antitumor activity is dependent on HER2 expression level rather than on HER2 amplification. Int $J$ Cancer. 2019; 145:3414-3424. doi:10.1002/ijc.32408

37. Shitara K, Bang Y-J, Iwasa S, et al. Trastuzumab deruxtecan in previously treated HER2-positive gastric cancer. $N$ Engl J Med. 2020;382:2419-2430. doi:10.1056/NEJMoa2004413

38. Stavenhagen JB, Gorlatov S, Tuaillon N, et al. Fc optimization of therapeutic antibodies enhances their ability to kill tumor cells in vitro and controls tumor expansion in vivo via low-affinity activating fcgamma receptors. Cancer Res. 2007;67:8882-8890. doi:10. 1158/0008-5472.CAN-07-0696

39. Catenacci DVT, Kang YK, Park H, et al.; CP-MGAH22-5 Study Group. Margetuximab plus pembrolizumab in patients with previously treated, HER2-positive gastro-oesophageal adenocarcinoma (CP-MGAH22-05): a single-arm, phase 1b-2 trial. Lancet Oncol. 2020;21(8):1066-1076. doi:10.1016/S1470-2045(20)30326-0 
40. Larkin J, Chiarion-Sileni V, Gonzalez R, et al. Combined nivolumab and ipilimumab or monotherapy in untreated melanoma. $N$ Engl J Med. 2015;373:23-34. doi:10.1056/NEJMoa1504030

41. Reck M, Rodríguez-Abreu D, Robinson AG, et al. Pembrolizumab versus chemotherapy for PD-L1-positive non-small-cell lung cancer. $N$ Engl J Med. 2016;375:1823-1833. doi:10.1056/NEJMoa 1606774

42. Motzer RJ, Escudier B, McDermott DF, et al. Nivolumab versus everolimus in advanced renal-cell carcinoma. $N$ Engl J Med. 2015;373:1803-1813. doi:10.1056/NEJMoa1510665

43. Bonotto M, Garattini SK, Basile D, et al. Immunotherapy for gastric cancers: emerging role and future perspectives. Expert Rev Clin Pharmacol. 2017;10:609-619. doi:10.1080/17512433.20 17.1313113

44. Fuchs CS, Doi T, Jang RW, et al. Safety and efficacy of pembrolizumab monotherapy in patients with previously treated advanced gastric and gastroesophageal junction cancer: phase 2 clinical KEYNOTE-059 trial. JAMA Oncol. 2018;4:e180013. doi:10.1001/ jamaoncol.2018.0013

45. Marabelle A, Le DT, Ascierto PA, et al. Efficacy of pembrolizumab in patients with noncolorectal high microsatellite instability/mismatch repair-deficient cancer: results from the phase II KEYNOTE-158 study. J Clin Oncol. 2020;38:1-10. doi:10.1200/JCO.19.02105

46. Muro K, Chung HC, Shankaran V, et al. Pembrolizumab for patients with PD-L1-positive advanced gastric cancer (KEYNOTE-012): a multicentre, open-label, phase $1 \mathrm{~b}$ trial. Lancet Oncol. 2016;17:717-726. doi:10.1016/S1470-2045(16)00175-3

47. Shitara K, Özgüroğlu M, Bang Y-J, et al. Pembrolizumab versus paclitaxel for previously treated, advanced gastric or gastro-oesophageal junction cancer (KEYNOTE-061): a randomised, open-label, controlled, phase 3 trial. Lancet. 2018;392:123-133. doi:10.1016/S0140-6736(18)31257-1

48. Kang Y-K, Boku N, Satoh T, et al. Nivolumab in patients with advanced gastric or gastro-oesophageal junction cancer refractory to, or intolerant of, at least two previous chemotherapy regimens (ONO-4538-12, ATTRACTION-2): a randomised, double-blind, placebo-controlled, phase 3 trial. Lancet. 2017;390:2461-2471. doi:10.1016/S0140-6736(17)31827-5

49. Janjigian YY, Bendell J, Calvo E, et al. CheckMate-032 study: efficacy and safety of nivolumab and nivolumab plus ipilimumab in patients with metastatic esophagogastric cancer. J Clin Oncol. 2018;36:2836-2844. doi:10.1200/JCO.2017.76.6212

50. Shitara K, Van Cutsem E, Bang Y-J, et al. Efficacy and safety of pembrolizumab or pembrolizumab plus chemotherapy vs chemotherapy alone for patients with first-line, advanced gastric cancer: the KEYNOTE-062 phase 3 randomized clinical trial. JAMA Oncol. 2020;6:1571-1580. doi:10.1001/jamaoncol.2020.3370

51. Wang F, Wei XL, Wang FH, et al. Safety, efficacy and tumor mutational burden as a biomarker of overall survival benefit in chemo-refractory gastric cancer treated with toripalimab, a PD-1 antibody in phase Ib/II clinical trial NCT02915432. Ann Oncol. 2019;30:1479-1486. doi:10.1093/annonc/mdz197

52. Bang Y-J, Ruiz EY, Van Cutsem E, et al. Phase III, randomised trial of avelumab versus physician's choice of chemotherapy as third-line treatment of patients with advanced gastric or gastrooesophageal junction cancer: primary analysis of JAVELIN gastric 300. Ann Oncol. 2018;29:2052-2060. doi:10.1093/annonc/ mdy 264

53. Kelly RJ, Lee J, Bang Y-J, et al. Safety and efficacy of durvalumab and tremelimumab alone or in combination in patients with advanced gastric and gastroesophageal junction adenocarcinoma. Clin Cancer Res. 2020;26:846-854. doi:10.1158/1078-0432.CCR19-2443

54. Chung HW, Lim J-B. Role of the tumor microenvironment in the pathogenesis of gastric carcinoma. World J Gastroenterol. 2014;20: 1667-1680. doi:10.3748/wjg.v20.i7.1667
55. Cassier PA, Italiano A, Gomez-Roca CA, et al. CSF1R inhibition with emactuzumab in locally advanced diffuse-type tenosynovial giant cell tumours of the soft tissue: a dose-escalation and dose-expansion phase 1 study. Lancet Oncol. 2015;16:949-956. doi:10.1016/S1470-2045(15)00132-1

56. Butowski N, Colman H, De Groot JF, et al. Orally administered colony stimulating factor 1 receptor inhibitor PLX3397 in recurrent glioblastoma: an Ivy foundation early phase clinical trials consortium phase II study. Neuro Oncol. 2016;18:557-564. doi:10.1093/neuonc/nov245

57. Pyonteck SM, Akkari L, Schuhmacher AJ, et al. CSF-1R inhibition alters macrophage polarization and blocks glioma progression. Nat Med. 2013;19:1264-1272. doi:10.1038/nm.3337

58. Chao J, Fuchs CS, Shitara K, et al. Assessment of pembrolizumab therapy for the treatment of microsatellite instability-high gastric or gastroesophageal junction cancer among patients in the KEYNOTE-059, KEYNOTE-061, and KEYNOTE-062 clinical trials. JAMA Oncol. 2021;7:895. doi:10.1001/jamaoncol.2021.0275

59. Jüttner S, Wissmann C, Jöns T, et al. Vascular endothelial growth factor-D and its receptor VEGFR-3: two novel independent prognostic markers in gastric adenocarcinoma. J Clin Oncol. 2006;24: 228-240. doi:10.1200/JCO.2004.00.3467

60. Ohtsu A, Shah MA, Van Cutsem E, et al. Bevacizumab in combination with chemotherapy as first-line therapy in advanced gastric cancer: a randomized, double-blind, placebo-controlled phase III study. J Clin Oncol. 2011;29:3968-3976. doi:10.1200/JCO.2011.36.2236

61. Shen L, Li J, Xu J, et al. Bevacizumab plus capecitabine and cisplatin in Chinese patients with inoperable locally advanced or metastatic gastric or gastroesophageal junction cancer: randomized, double-blind, phase III study (AVATAR study). Gastric Cancer. 2015;18:168-176. doi:10.1007/s10120-014-0351-5

62. Cunningham D, Stenning SP, Smyth EC, et al. Peri-operative chemotherapy with or without bevacizumab in operable oesophagogastric adenocarcinoma (UK Medical Research Council ST03): primary analysis results of a multicentre, open-label, randomised phase 2-3 trial. Lancet Oncol. 2017;18:357-370. doi:10.1016/S1470-2045(17) 30043-8

63. Ma J, Yao S, Li X-S, Kang H-R, Yao -F-F, Du N. Neoadjuvant therapy of DOF regimen plus bevacizumab can increase surgical resection ratein locally advanced gastric cancer: a randomized, controlled study. Medicine (Baltimore). 2015;94:e1489. doi:10.1097/ MD.0000000000001489

64. Aprile G, Bonotto M, Ongaro E, Pozzo C, Giuliani F. Critical appraisal of ramucirumab (IMC-1121B) for cancer treatment: from benchside to clinical use. Drugs. 2013;73:2003-2015. doi:10.1007/ s40265-013-0154-8

65. Fuchs CS, Shitara K, Di Bartolomeo M, et al. Ramucirumab with cisplatin and fluoropyrimidine as first-line therapy in patients with metastatic gastric or junctional adenocarcinoma (RAINFALL): a double-blind, randomised, placebo-controlled, phase 3 trial. Lancet Oncol. 2019;20:420-435. doi:10.1016/S1470-2045(18)30791-5

66. Li J, Qin S, Xu J, et al. Randomized, double-blind, placebo-controlled phase III trial of apatinib in patients with chemotherapy-refractory advanced or metastatic adenocarcinoma of the stomach or gastroesophageal junction. $J$ Clin Oncol. 2016;34:1448-1454. doi:10.1200/JCO.2015.63.5995

67. Pavlakis N, Sjoquist KM, Martin AJ, et al. Regorafenib for the treatment of advanced gastric cancer (INTEGRATE): a multinational placebo-controlled phase II trial. J Clin Oncol. 2016;34:2728-2735. doi:10.1200/JCO.2015.65.1901

68. Shan F, Miao R, Xue K, et al. Controlling angiogenesis in gastric cancer: a systematic review of anti-angiogenic trials. Cancer Lett. 2016;380:598-607. doi:10.1016/j.canlet.2015.12.023

69. Sahin U, Koslowski M, Dhaene K, et al. Claudin-18 splice variant 2 is a pan-cancer target suitable for therapeutic antibody development. Clin Cancer Res. 2008;14:7624-7634. doi:10.1158/1078-0432.CCR08-1547 
70. Sahin U, Schuler M, Richly H, et al. A phase I dose-escalation study of IMAB362 (Zolbetuximab) in patients with advanced gastric and gastro-oesophageal junction cancer. Eur J Cancer. 2018;100:17-26. doi:10.1016/j.ejca.2018.05.007

71. Türeci O, Sahin U, Schulze-Bergkamen H, et al. A multicentre, phase IIa study of zolbetuximab as a single agent in patients with recurrent or refractory advanced adenocarcinoma of the stomach or lower oesophagus: the MONO study. Ann Oncol. 2019;30:1487-1495. doi:10.1093/annonc/mdz199

72. Sahin U, Tureci Ö, Manikhas GM, et al. Zolbetuximab combined with EOX as first-line therapy in advanced CLDN18.2+ gastric (G) and gastroesophageal junction (GEJ) adenocarcinoma: updated results from the FAST trial. $J$ Clin Oncol. 2019;37:16. doi:10.1200/ JCO.2019.37.4_suppl.16

73. Al-Batran S-E, Schuler MH, Zvirbule Z, et al. FAST: an international, multicenter, randomized, phase II trial of epirubicin, oxaliplatin, and capecitabine (EOX) with or without IMAB362, a first-inclass anti-CLDN18.2 antibody, as first-line therapy in patients with advanced CLDN18.2+ gastric and gastroesophageal junction (GEJ) adenocarcinoma. J Clin Oncol. 2016;34:LBA4001. doi:10.1200/ JCO.2016.34.18 suppl.LBA4001

74. Pearson A, Smyth E, Babina IS, et al. High-level clonal FGFR amplification and response to FGFR inhibition in a translational clinical trial. Cancer Discov. 2016;6:838-851. doi:10.1158/21598290.CD-15-1246

75. Dienstmann R, Rodon J, Prat A, et al. Genomic aberrations in the FGFR pathway: opportunities for targeted therapies in solid tumors. Ann Oncol. 2014;25:552-563. doi:10.1093/annonc/mdt419

76. Babina IS, Turner NC. Advances and challenges in targeting FGFR signalling in cancer. Nat Rev Cancer. 2017;17:318-332. doi:10.1038/ nrc. 2017.8

77. Catenacci DV, Tesfaye A, Tejani M, et al. Bemarituzumab with modified FOLFOX6 for advanced FGFR2-positive gastroesophageal cancer: FIGHT phase III study design. Future Oncol. 2019;15:2073-2082. doi:10.2217/fon-2019-0141

78. Dienstmann R, Mason MJ, Sinicrope FA, et al. Prediction of overall survival in stage II and III colon cancer beyond TNM system: a retrospective, pooled biomarker study. Ann Oncol. 2017;28:1023-1031. doi:10.1093/annonc/mdx052

79. Gambardella V, Fleitas T, Cervantes A. Understanding mechanisms of primary resistance to checkpoint inhibitors will lead to precision immunotherapy of advanced gastric cancer. Ann Oncol. 2019;30:351-352. doi:10.1093/annonc/mdz008
80. Van Cutsem E, Bang Y-J, Mansoor W, et al. A randomized, open-label study of the efficacy and safety of AZD4547 monotherapy versus paclitaxel for the treatment of advanced gastric adenocarcinoma with FGFR2 polysomy or gene amplification. Ann Oncol. 2017;28:1316-1324. doi:10.1093/annonc/mdx107

81. Wainberg ZA, Enzinger PC, Kang Y-K, et al. Randomized double-blind placebo-controlled phase 2 study of bemarituzumab combined with modified FOLFOX6 (MFOLFOX6) in first-line (1L) treatment of advanced gastric/gastroesophageal junction adenocarcinoma (FIGHT). $J$ Clin Oncol. 2021;39:160. doi:10.1200/ JCO.2021.39.3_suppl.160

82. Kim HS, Kim MA, Hodgson D, et al. Concordance of ATM (Ataxia Telangiectasia Mutated) immunohistochemistry between biopsy or metastatic tumor samples and primary tumors in gastric cancer patients. Pathobiol J Immunopathol Mol Cell Biol. 2013;80:127-137. doi:10.1159/000346034

83. Bang Y-J, Xu R-H, Chin K, et al. Olaparib in combination with paclitaxel in patients with advanced gastric cancer who have progressed following first-line therapy (GOLD): a double-blind, randomised, placebo-controlled, phase 3 trial. Lancet Oncol. 2017;18:1637-1651. doi:10.1016/S1470-2045(17)30682-4

84. Malka D, François E, Penault-Llorca F, et al. FOLFOX alone or combined with rilotumumab or panitumumab as first-line treatment for patients with advanced gastroesophageal adenocarcinoma (PRODIGE 17-ACCORD 20-MEGA): a randomised, open-label, three-arm phase II trial. Eur $J$ Cancer. 2019;115:97-106. doi:10.1016/j.ejca.2019.04.020

85. Waddell T, Chau I, Cunningham D, et al. Epirubicin, oxaliplatin, and capecitabine with or without panitumumab for patients with previously untreated advanced oesophagogastric cancer (REAL3): a randomised, open-label phase 3 trial. Lancet Oncol. 2013;14:481-489. doi:10.1016/S1470-2045(13)70096-2

86. Lordick F, Kang Y-K, Chung H-C, et al. Capecitabine and cisplatin with or without cetuximab for patients with previously untreated advanced gastric cancer (EXPAND): a randomised, open-label phase 3 trial. Lancet Oncol. 2013;14:490-499. doi:10.1016/S14702045(13)70102-5

87. Corso S, Pietrantonio F, Apicella M, et al. Optimized EGFR blockade strategies in EGFR addicted gastroesophageal adenocarcinomas. Clin Cancer Res. 2021;27(11):3126-3140. doi:10.1158/1078-0432.CCR20-0121

\section{Publish your work in this journal}

Biologics: Targets and Therapy is an international, peer-reviewed journal focusing on the patho-physiological rationale for and clinical application of Biologic agents in the management of autoimmune diseases, cancers or other pathologies where a molecular target can be identified. This journal is indexed on PubMed Central, CAS, EMBase,
Scopus and the Elsevier Bibliographic databases. The manuscript management system is completely online and includes a very quick and fair peer-review system, which is all easy to use. Visit $\mathrm{http}: / /$ www.dovepress.com/testimonials.php to read real quotes from published authors. 